BMJ Open

Diabetes

Research

\& Care

\title{
Diabetes causal attributions among affected and unaffected individuals
}

\author{
Margaret K Rose, ${ }^{1}$ Kristi A Costabile, ${ }^{2}$ Sarah E Boland, ${ }^{1}$ Rachel W Cohen, ${ }^{1}$ \\ Susan Persky ${ }^{\odot}$
}

To cite: Rose MK, Costabile KA, Boland SE, et al. Diabetes causal attributions among affected and unaffected individuals. BMJ Open Diab Res Care 2019;7:e000708. doi:10.1136/ bmjdrc-2019-000708

Received 18 June 2019 Revised 23 July 2019 Accepted 13 August 2019
Check for updates

\section{(C) Author(s) (or their} employer(s)) 2019. Re-use permitted under CC BY-NC. No commercial re-use. See rights and permissions. Published by BMJ.

${ }^{1}$ Social and Behavioral Research Branch, National Human Genome Research Institute, Bethesda, Maryland, USA

${ }^{2}$ Department of Psychology, lowa State University, Ames, lowa, USA

Correspondence to Dr Susan Persky; perskys@mail.nih.gov

\section{ABSTRACT}

Objective The present study aims to describe and compare causal attributions for type 1 diabetes (T1D) and type 2 diabetes (T2D) among affected and unaffected individuals and to investigate the relationships among attributions, attitudes, and beliefs.

Research design and methods Adults with no diabetes ( $\mathrm{N}=458)$, T1D ( $\mathrm{N}=192)$, or T2D ( $\mathrm{N}=207)$ completed an online survey. Measures assessed diabetes conceptual knowledge, causal attributions for T1D and T2D, perceived control over diabetes onset, and favorability judgements of individuals affected by each type.

Results Results indicate general agreement on causal attributions for T1D and T2D among all respondent groups, with some divergences by disease status. All respondents attributed both T1D and T2D to genetics, and genetic attributions were positively associated with favorability judgements of individuals with $\mathrm{T} 2 \mathrm{D}$, but not those with T1D.

Conclusions This report sets the stage for investigations into how and why attributions for T1D and T2D differ and the implications of these differences including stigmatization of individuals with diabetes and diabetesrelated self-concept. Additionally, this work can inform efforts towards clinical and public health education to prevent and optimize treatment of T1D and T2D.

\section{INTRODUCTION}

Type 1 diabetes (T1D) and type 2 diabetes (T2D) have different pathophysiology, different treatment approaches, and different complements of causal, or risk, factors. The way in which individuals understand these casual factors has considerable power to shape attitudes and beliefs about the disease, about disease management, and about individuals affected by it. ${ }^{12}$ Because of the potential power of causal attributions, and the dearth of data in this domain, the current report focuses on patterns of causal attributions among individuals with T1D, T2D, and those who are unaffected.

\section{Diabetes risk factors}

T1D and T2D are both multifactorial in their causes; however, mechanisms between risk factors and disease onset are not well understood. Estimates of genetic influence on the development of both T1D and T2D

\section{Significance of this study}

What is already known about this subject?

- Causal attributions play an important role in the formation of attitudes towards health conditions and individuals with such conditions; however, this has not been explicitly studied in diabetes.

What are the new findings?

- All individuals, regardless of diabetes status, follow similar patterns in their causal attributions for diabetes, with important divergences including beliefs about the role of germs/viruses versus genetics in type 1 diabetes.

- This work establishes relationships between perceived control over diabetes onset and favorability of individuals with diabetes and the endorsement of causal factors including: diet, physical activity, and overweight.

- On the flip side, there was not consistent evidence of expected relationships with genetic attributions including low controllability beliefs and increased favorability of affected individuals.

How might these results change the focus of research or clinical practice?

- The current findings challenge assumptions made in the diabetes literature. Future research must incorporate the notion that that causal attributions for diabetes are inconsistent among groups of affected and unaffected individuals and that causal attributions for diabetes that appear low-control (eg, genetics and chance) are not reliably tied to low control beliefs among individuals with diabetes.

vary widely. T1D is thought to arise through a combination of genetic and environmental risk factors. ${ }^{3}{ }^{4}$ Overall, literature suggests that T1D is highly heritable, although the complex genetic nature of the disease does not allow for an exact risk measurement. The nature of the environmental insult is unknown; however, one hypothesis involves viral exposure. ${ }^{5}$

Risk factors for T2D include genetic and environmental components as well. ${ }^{6}$ T2D is highly heritable; however, the range of estimates of heritability is large and varies as a function of the sample and length of study. ${ }^{78}$ 
Risk factors for T2D also include lifestyle factors such as smoking, diet, and physical activity levels. ${ }^{9}$ When actions are taken to mitigate these risk factors, T2D can be prevented in some, though not all, individuals. ${ }^{10}$

\section{Causal attributions}

Weiner's ${ }^{11}$ classic work on causal attributions suggests that perceived causes of a condition can affect attitudes, emotions, beliefs, and behaviors directed toward those affected with that condition. This theory may have important implications for diabetes due, in part, to differences in perceptions of T1D and T2D. To the extent that the cause of a condition is perceived to be internal, unstable, and controllable, this should lead to increased blame. The relationship between attributions and blame has been studied in other illness domains including mental illness and obesity. ${ }^{12}{ }^{13}$ Within obesity, which is closely tied to T2D, beliefs pertaining to the control over obesity (or the extent to which obesity is perceived to have been affected by actions under the individual's control) were a reliable predictor of dislike of people with overweight. ${ }^{14}$ The corollary is that to the extent obesity is perceived to be out of the control of an individual, the less individuals will tend to be disliked. ${ }^{15}$

\section{Attitudes toward individuals affected by diabetes}

There is increasing evidence indicating that individuals, both with T1D and T2D, are negatively judged due to their condition. Individuals with T2D report feeling blamed by others due to the assumptions that T2D is behavioral in nature. ${ }^{16}$ This may be due in part to the salience of behavioral factors (ie, diet and physical activity) in T2D management and prevention, which likely privileges these causal factors in conceptions of diabetes etiology.

Individuals with T1D also report feeling negatively judged, but often report that this occurs through misplaced blame meant for individuals with T2D. ${ }^{17}$ For example, media coverage of diabetes is dominated by T2D compared with T1D or is often not specified as to which type of diabetes is discussed. ${ }^{18}$ These communications likely shape the beliefs that unaffected individuals (those without a diabetes diagnosis of either type), and likely some affected individuals, have about diabetes and the role of personal responsibility in disease onset.

\section{Causal ttributions and controllability beliefs}

The literature on causal attributions in diabetes is limited. Much of the pertinent work focuses on beliefs individuals hold about T2D or fails to specify the diabetes type of interest. Furthermore, responses of each diabetes status group (unaffected, T1D, or T2D) tend to be studied in isolation. Relevant studies have found that when participants are asked about T2D, they tend to make causal attributions that include both genetic and behavioral factors, rather than choosing one or the other. ${ }^{19-21}$ However, in one instance when unaffected participants were asked to compare the causes of T1D and T2D, they were more likely to attribute behavioral factors to T2D and genetic factors to T1D. ${ }^{22}$

It is often assumed that behavioral risk factors, like diet and physical activity levels, are perceived to be under the control of the individual and thus associated with high controllability beliefs about disease onset and that genetic explanations are associated with low controllability beliefs. However, this assumption is rarely tested and has never been assessed in the context of diabetes. The relationship between perceived control over diabetes onset and causal attributions must be established in this domain to fully understand the role of attributions play in informing attitudes, beliefs, and behavior toward individuals with diabetes.

\section{The influence of disease status}

There are no known comparisons of diabetes causal attributions from the perspective of individuals with and without diabetes. It is thus unknown whether or how the lived experience of individuals with diabetes influences their perception of what causes their own disease. Furthermore, it is also unknown whether living with one type of diabetes influences attributions related to the other type of diabetes. Negative health outcomes have been shown to arise when individuals feel blamed or blame themselves for causing their condition. ${ }^{23-25}$ As such, the manner with which individuals with T1D and T2D understand and attribute causes to their own diagnosis has potential implications for identity, diabetes management, and care-seeking. This is also relevant for unaffected individuals' willingness to engage in preventative measures to reduce risk of T2D. Establishing comparative patterns of causal understanding could inform decisions about educational content in public health messages aimed at various groups. Such data may also highlight areas of educational need and gaps in causal understanding.

\section{Diabetes knowledge}

There is likely large variability in diabetes knowledge among individuals with T1D, with T2D, and unaffected individuals. Clearly, individuals with diabetes are expected to be more knowledgeable about their condition than unaffected individuals. Often, a new diagnosis of either type is accompanied with an educational session to convey practical information related to daily management of diabetes. Literature reporting on diabetes knowledge focuses on this practical knowledge, and consequently there is not information on conceptual knowledge (eg, pertaining to the causal factors, pathophysiology, treatments and outcomes of diabetes) levels among the different groups.

\section{The current study}

The aims of this study are: (1) to describe causal attributions for T1D and T2D among individuals with and without a diabetes diagnosis, (2) to assess the relationship between attributions and perceived control over diabetes onset, and (3) to analyze associations between 
causal attributions and favorability judgements of individuals affected by T1D and T2D. Favorability assesses general, all-encompassing feelings of positivity or negativity toward individuals. ${ }^{26}$ These feelings are not tied to beliefs specific to any one condition, allowing comparisons to be assessed across groups that do not share many commonalities, like T1D and T2D. We also begin our analysis by assessing diabetes knowledge, as this is a necessary precondition for holding distinct and meaningful attributions for the two disease types.

First, we hypothesized that respondents in all groups would indicate higher genetic attributions for T1D than T2D and higher behavioral (diet and physical activity) attributions for T2D than for T1D. We also hypothesized that genetic attributions for T2D would be highest among those with the condition, whereas genetic attributions for T1D would be high among all respondent groups. Although we expected variability, we did not lay out a priori hypotheses regarding other causal attribution levels. For both T1D and T2D, we hypothesized that behavioral factors would be positively related to perceived control over T1D and T2D onset, whereas non-behavioral factors (eg, genetics) would be negatively related to perceived control. Finally, we hypothesized that, for T2D, genetic attributions would be positively related to favorability judgements of T2D and that behavioral attributions would be negatively related to favorability judgements of this group, similarly to obesity. We did not make hypotheses of this nature about T1D given the lack of literature to draw from.

\section{METHOD}

\section{Participants}

Data for this report were drawn from the Diabetes, Identity, Attributions, and Health Study, which examines individuals' beliefs about the causes of diabetes and how those beliefs relate to social identity, health behavior, and overall health. Eligible participants for the study included adults, 18 years of age or older, who fell into one of the following three categories: unaffected (no diabetes diagnosis), diagnosed with T1D, or diagnosed with T2D. Unaffected participants $(n=458)$ were recruited through Amazon Mechanical Turk (mTurk) and were compensated \$1.45. Participants who self-reported a T1D or T2D diagnosis were recruited through both ResearchMatch (a web-based clinical research recruitment registry) and Facebook (through established groups for affected individuals). Participants with T1D $(n=192)$ and T2D $(n=207)$ completed a longer survey than unaffected participants and were compensated $\$ 10.00$ for their time.

\section{Procedure}

Participants completed an online, anonymous survey administered via SurveyMonkey. Unaffected respondents and those with a diabetes diagnosis completed identical measures assessing their attitudes and beliefs regarding T1D and T2D in a counterbalanced order. Participants with diabetes then completed additional measures specific to their experience with their diagnosed type. For purposes of data quality, all participants were asked to commit to not using outside sources when answering survey questions and to confirm their diabetes status (without penalty for initial misrepresentation) at the end. Participants were excluded from analysis if they did not fulfil data quality criteria. Participants were also excluded if they did not have sufficient knowledge of diabetes (see below).

\section{Measures}

The measures reported below were administered to all three respondent groups.

Diabetes knowledge: to assess sufficient knowledge about the difference between T1D and T2D, open-ended questions were presented wherein participants were asked to explain, in their own words, what T1D and T2D are and the difference between the two.

Causal attributions: attributions about eight causal factors were assessed for T1D and T2D: diet, physical activity, overweight, environment, family environment, genetics, germ/virus, and chance. Participants were asked to respond to the following question on a 7-point Likert scale $(1=$ strongly disagree to $7=$ strongly agree $)$ : 'Indicate the extent to which you agree or disagree that each of the following factors cause or contribute to a persons' risk for getting [T1D/T2D] sometime in his/her lifetime. ${ }^{27} 28$

Control: participants were asked to indicate if they agreed that a person could control whether they developed T1D and T2D, via two closed-ended questions for each type of diabetes on a five-point scale (1=strongly disagree to $5=$ strongly agree; $\alpha=0.800$ for T1D control and $\alpha=0.724$ for T2D control). ${ }^{27}$

Favorability judgements: participants were asked to indicate their perceived favorability of individuals diagnosed with T1D, and separately for T2D, via one closed-ended question on a nine-point scale ( $1=n o t$ very favorable to $9=$ very favorable $).{ }^{26}$

\section{Analysis}

Coding of diabetes knowledge: participants' conceptual diabetes knowledge responses were coded on eight dimensions for which the two types of diabetes differ. Two trained coders achieved coding agreement (kappa levels ranging from 0.72 to 1.00$)$. The dimensions were: age of onset, casual factors, severity, prevalence, symptom controllability, pathophysiology, treatment, and outcome. Explanations of the dimensions are provided in online supplementary table 1 . Three understanding levels were established based on the number of dimensions an individual described completely and correctly. An individual was classified as having a good understanding if they mentioned two or more dimensions and made no incorrect statements. An $o k$ understanding was defined as only mentioning one dimension or more than one dimension with minor incorrect statements. Understanding was classified as poor when the participant failed to mention a 
Table 1 Sample characteristics and descriptive statistics for respondents included in analyses

\begin{tabular}{lcccc}
\hline Variable & $\begin{array}{l}\text { Unaffected } \\
(\mathbf{n}=\mathbf{3 2 0})\end{array}$ & $\begin{array}{l}\text { T1D } \\
(\mathbf{n}=\mathbf{1 8 2})\end{array}$ & $\begin{array}{l}\text { T2D } \\
(\mathbf{n}=1 \mathbf{1 9 0})\end{array}$ & P value * \\
\hline Age, years & $34.2(9.5)$ & $40.4(14.3)$ & $54.0(11.5)$ & $<0.001$ \\
\hline BMI & $27.1(6.8)$ & $26.6(5.8)$ & $33.5(7.8)$ & $<0.001$ \\
\hline College graduate & $160(50.0 \%)$ & $126(69.2 \%)$ & $102(53.7 \%)$ & $<0.001$ \\
\hline Non-Hispanic white & $159(49.7 \%)$ & $138(76.2 \%)$ & $135(71.1 \%)$ & $<0.001$ \\
\hline Female & $237(74.5 \%)$ & $160(89.4 \%)$ & $149(79.7 \%)$ & $<0.001$ \\
\hline
\end{tabular}

$M(S D)$ or frequency (\%) reported.

${ }^{*} \mathrm{P}$ value reflects group differences among respondent group status.

BMI, body mass index; T1D, type 1 diabetes; T2D, type 2 diabetes.

full dimension (ie, only remarked on one type) or made major incorrect statements in their responses. Responses that only mentioned 'age of onset' were classified as poor because T1D was already referred to as 'juvenile' diabetes in the questionnaire. Participants with a poor understanding of the difference between T1D and T2D were excluded from further analysis. Examples of responses and their classifications are provided in online supplementary table 2 .

\section{Data analysis}

Analyses of covariance (ANCOVAs) were conducted to assess differences among respondent groups for each causal factor regarding both T1D and T2D, as well as differences for favorability judgements of individuals with T1D and T2D. Pairwise comparisons assessed attribution and favorability responses between individual respondent groups. Regressions were conducted to determine the association between causal attributions and perceived control, and causal attributions and favorability. The Benjamini-Hochberg Procedure was used to control for the false discovery rate that may arise due to these multiple comparisons. ${ }^{29}$ Covariates included in the ANCOVAs and regressions were age, gender, race (non-Hispanic white or not), education (college graduate or not), and body mass index as these differed between respondent groups.

\section{RESULTS}

\section{Descriptive statistics}

Online supplementary table 3 displays the demographics of respondents in all understanding groups. The majority of participants in all three respondent categories had $o k$ or good understanding of the difference between T1D and T2D. Few participants with either a T1D or T2D diagnosis had an understanding classified as poor.

One hundred and sixty-five individuals with poor understanding were excluded from further analysis. Ten additional participants were excluded due to clear plagiarism in their response to the knowledge items. Six hundred and ninety-two individuals with $o k$ and good classifications of understanding were combined and included in further analysis. Demographic information for these individuals is included in table 1.

\section{Diabetes knowledge}

The percentage of participants that mentioned each of the eight dimensions is displayed in figure 1. Overall, participants were most likely to mention onset, causal factors, and treatment; however this pattern varied by respondent diabetes status, $F(14,1368)=23.17$, $\mathrm{p}<0.001$.

\section{Causal attributions}

The mean causal attributions for T1D are shown in figure 1 and table 2. In general, all respondent groups

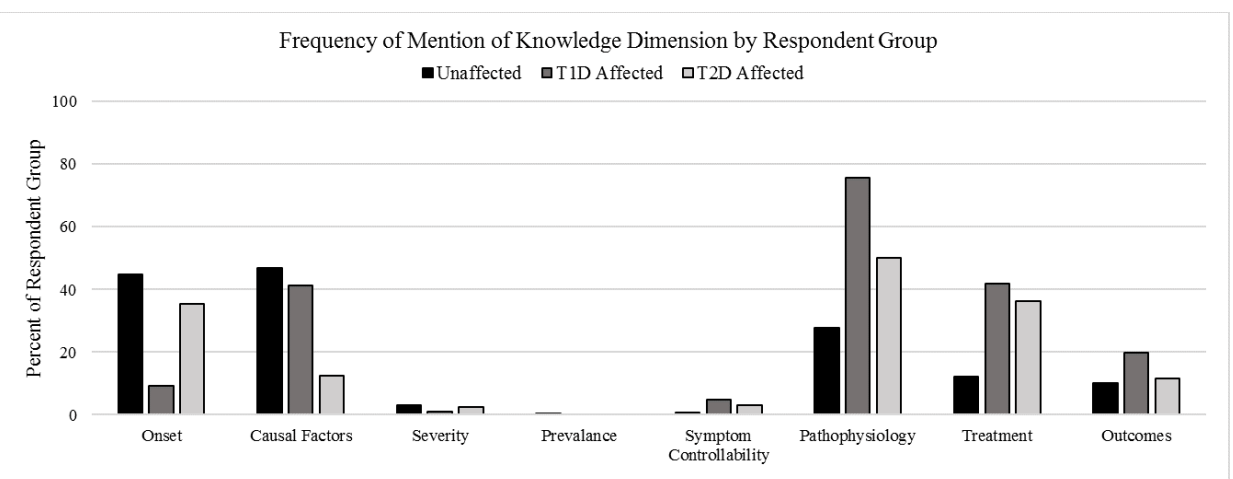

Figure 1 Frequency of mention of the eight dimensions of understanding from those with sufficient understanding. T1D, type 1 diabetes; T2D, type 2 diabetes. 


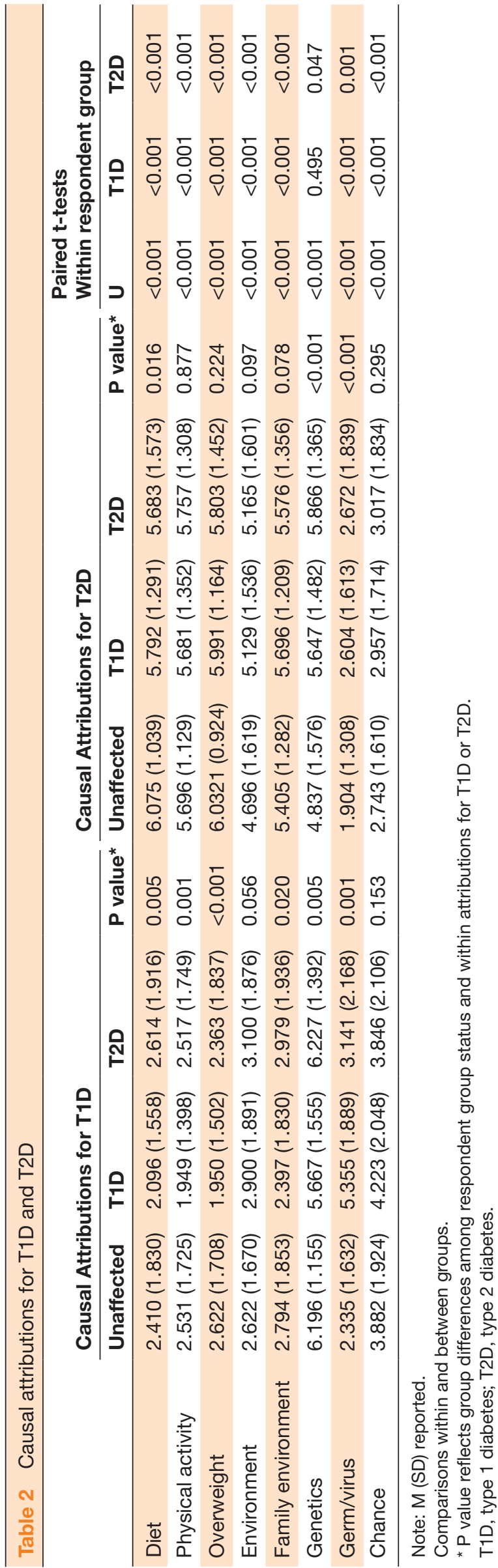

disagreed (ie, had means below the scale midpoint) that the following factors could be implicated in the development of the disease: overweight, diet, physical activity, environment, and family environment. Unaffected respondents, and those with T2D, had similar causal attributions for T1D, which tended to differ from respondents with T1D. Participants with T1D attributed a germ or virus as a cause of T1D at significantly higher levels than unaffected participants and those with T2D. Participants with T1D also made significantly lower attributions to overweight, diet, physical activity, family environment, and genetics for causing T1D than the other two respondent groups.

Figure 2 and table 2 show causal attributions for T2D. General alignment between the three groups was high here as well. All groups agreed that the following six out of the eight factors could cause or contribute to T2D: overweight, diet, physical activity, environment, family environment, and genetics. Unaffected individuals demonstrated significantly lower endorsement of genetic, environmental, and germ/virus factors and attributed diet more highly as a cause of T2D than the affected groups.

Table 2 compares means for causal attributions between T1D and T2D, within each respondent group. Unaffected individuals, and those with T2D, attributed genetics as a cause of T1D more than they did for T2D. Respondents with T1D equally attributed genetics as a cause of T1D and T2D. All groups significantly differed in their attributions of all other factors to T1D versus T2D.

\section{Control}

Figure 3 shows perceived control over T1D and T2D onset, as well as associations between causal attributions and perceived control over the onset of T1D and T2D. Across all respondent groups, the mean perceived control over $\mathrm{T} 1 \mathrm{D}$ onset $(\mathrm{M}=1.38, \mathrm{SD}=0.94)$ was significantly lower than the mean perceived control over T2D onset $(\mathrm{M}=3.98, \mathrm{SD}=0.81) ; t(684)=-48.26, \mathrm{p}<0.001$. There was a significant effect of respondent diabetes status on perceived control over T1D and T2D onset, $F(2,663)=6.09, \mathrm{p}=0.018$. For all respondent groups, higher levels of perceived control were associated with the following casual factors: diet, physical activity, and overweight. Genetics, germ/virus, and chance were associated with lower levels of perceived control in some cases; however, relationships varied by respondent status and diabetes type.

\section{Favorability}

Figure 4 shows favorability levels of individuals with T1D and T2D as well as the associations between these levels and casual attributions. Across all respondent groups, the mean favorability level of individuals with T1D $(M=6.90$, $S D=1.91)$ was significantly higher than the mean favorability of individuals with T2D $(M=5.72, \quad S D=2.03)$; $t(685)=14.04, \mathrm{p}<0.001$. There was a significant effect of respondent diabetes status on favorability judgements of 


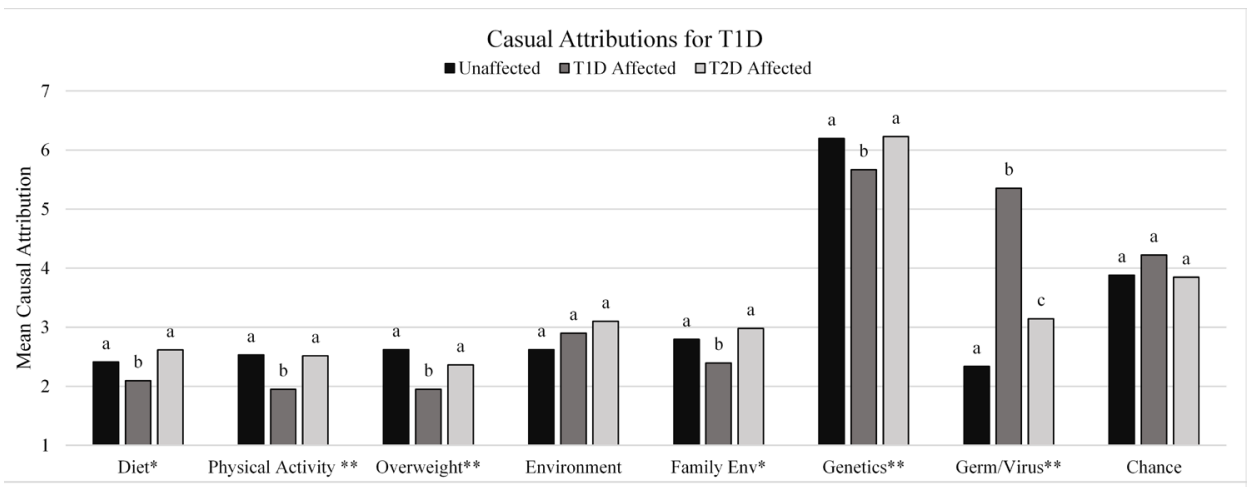

Causal Attributions for T2D

-Unaffected $\quad$ QT1D Affected $\quad$ 口T2D Affected

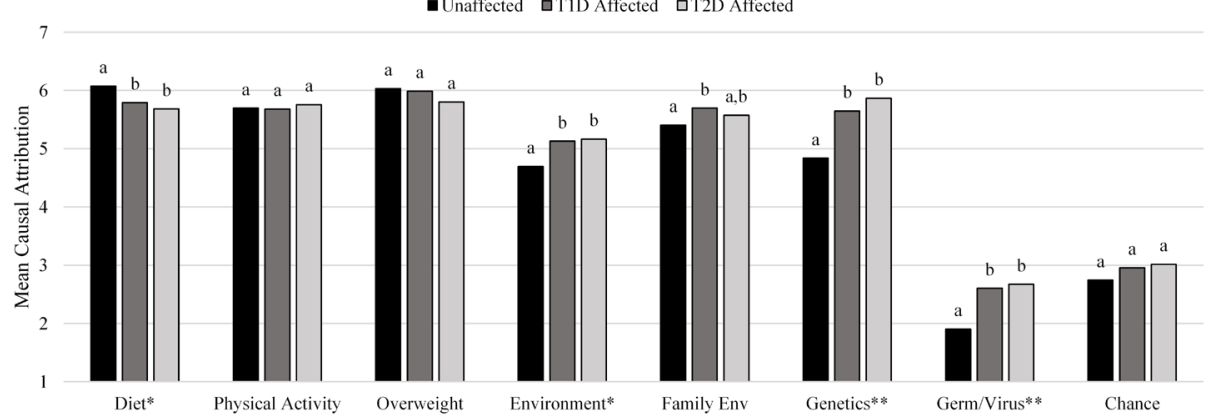

Figure 2 Causal attributions for T1D and T2D. ${ }^{*} \mathrm{P}<.05$, ${ }^{* *} \mathrm{P}<.01$ by factor. Bars that share a letter within each factor are not significantly different from one another at $p<.05$. T1D, type 1 diabetes; T2D, type 2 diabetes.

individuals with T1D and T2D, $F(2,664)=9.79$, p $<0.001$. Moreover, across all respondent groups, the degree to which an individual identified overweight or a behavioral factor as a cause of diabetes (diet and physical activity) was negatively associated with the favorability ratings of both individuals with T1D and those with T2D. Uncontrollable factors (genetics, germ/virus, and chance) were positively associated with favorability of individuals with T2D, among all respondent groups.

\section{DISCUSSION}

The present report examined how diabetes knowledge, causal attributions, control beliefs, and favorability

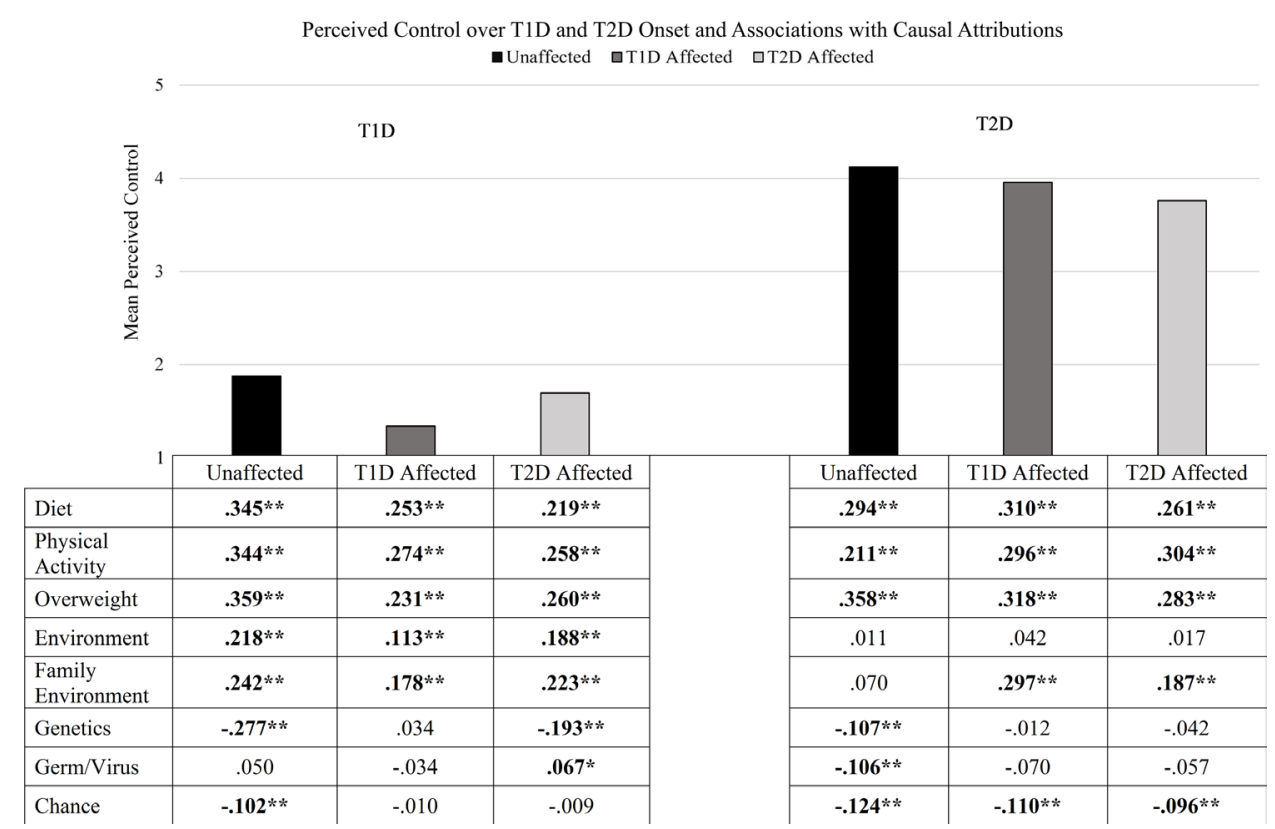

Figure 3 Perceived control over diabetes onset means (graph, scale 1-5) and associations between causal factor and perceived control (table, unstandardized $B$ values). ${ }^{*} P<.05 .{ }^{* *} \mathrm{p}<.01$ by row, within T1D or T2D. Bars that share a letter within each diabetes type are not significantly different from one another at $p<.05$. T1D, type 1 diabetes; T2D, type 2 diabetes. 


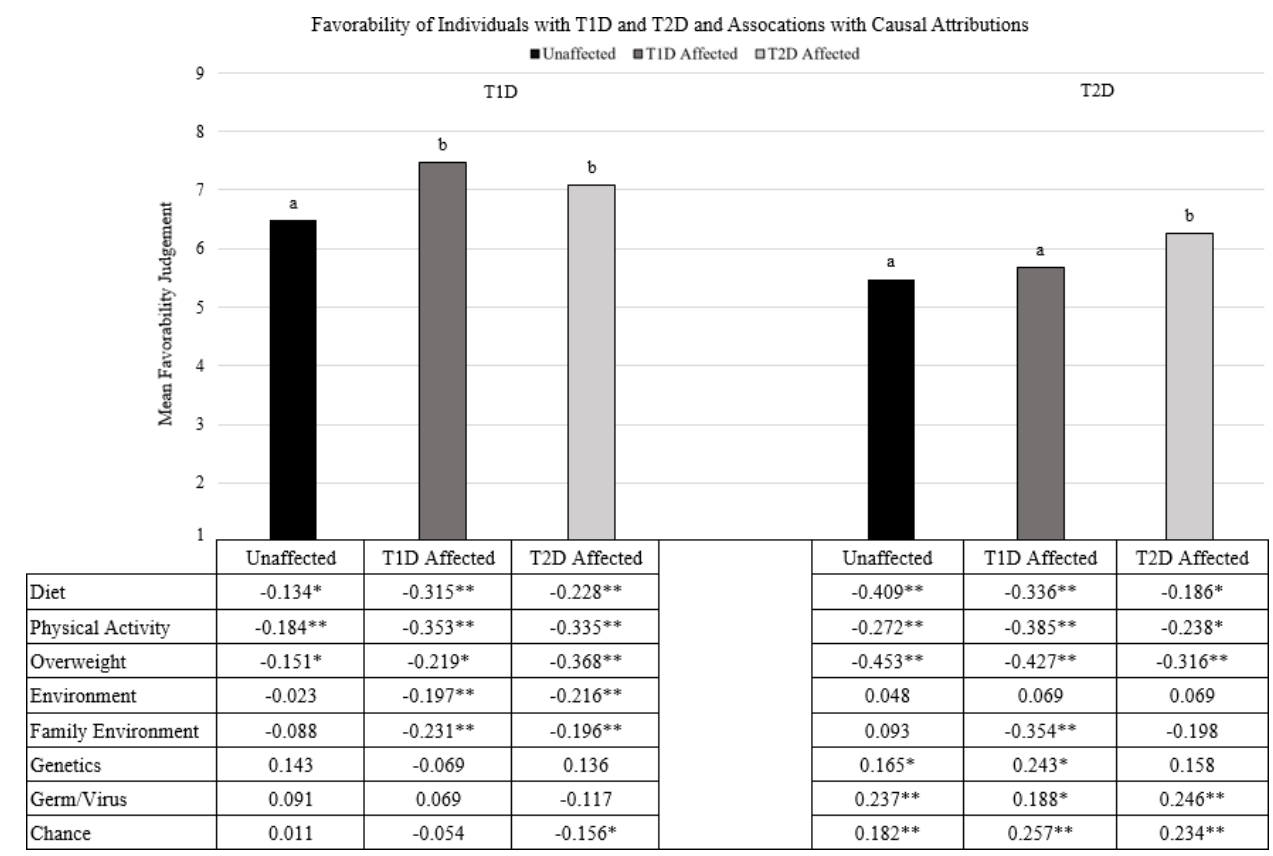

Figure 4 Favorability means (graph, scale 1-9) and associations between causal factor and favorability (table, unstandardized $B$ values). ${ }^{*} \mathrm{P}<.05$. ${ }^{* *} \mathrm{p}<.01$ by row, within T1D or T2D. Bars that share a letter within each diabetes type are not significantly different from one another at $\mathrm{p}<.05$. T1D, type 1 diabetes; T2D, type 2 diabetes.

judgements varied as a function of respondent diabetes status and how causal attributions related to control beliefs and favorability judgements. Results indicated general agreement as to causal attributions for T1D and T2D among individuals with and without diabetes. As expected, behavioral causal factors (diet and physical activity), as well as overweight, were generally attributed as causes for T2D, but not T1D. Consistent with the literature, ${ }^{24} 27$ genetics was attributed as a cause for both T1D and T2D. Although, when genetic attributions were compared, attributions of unaffected respondents and those with T2D were significantly higher for T1D than for T2D, in line with hypotheses; individuals with T1D made similar genetic attributions for both T1D and T2D.

\section{Respondents' diabetes status and attributions}

Including participants with and without diabetes in the current study allowed for between-group comparisons not previously possible. Group-based comparisons suggest that the experience of living with T1D or T2D influences the causal attributions made for one's own disease. The most notable difference among individuals with T1D was in their endorsement of a germ or virus as a cause of T1D. Despite the lack of a specific germ or virus being implicated as the proximal cause of T1D, viral influence is widely hypothesized. ${ }^{5}$ It is sensible that affected individuals would be more familiar with the science in this domain. Interestingly, respondents with T1D had lower attributions to genetics for T1D than the other groups. This is inconsistent with general research on self-serving bias, in which an individual affected by T1D might stress the uncontrollable factors associated with their own disease type and likewise stress the controllable factors associated with T2D. ${ }^{30}$ Although this finding was not predicted, relatively lower attribution of genetics for T1D may occur because many individuals diagnosed with T1D may not observe T1D clustering in their families. ${ }^{31}$ Overall, these differences suggest that attributions are informed by experiences unique to individuals with T1D.

A difference in affected participants' causal attributions is also seen for T2D. Unaffected respondents attributed genetics as a cause of T2D significantly less than affected groups. It may be less apparent to individuals who do not live with diabetes that a non-behavioral factor like genetics can play a large role in risk for T2D.

It has not been explicitly studied whether living with one type of diabetes influences attributions for another type of diabetes. Results suggest a knowledge asymmetry in that individuals with T1D have causal attributions for T2D that are similar to those living with the condition while the reverse is not true among individuals with T2D. T1D is most commonly diagnosed in childhood and so participants with T1D have likely had their diagnoses longer than those with T2D. Additionally, T1D may be a more central part of an affected individual's life and identity due to disease severity. This may result in a better understanding of diabetes as a whole. Additionally, knowledge about T2D may be useful for individuals with T1D when explaining the difference between the types to correct misconceptions and possibly to mitigate misplaced blame and stigma.

\section{Controllability beliefs}

While attributions for behavioral factors and overweight were associated with higher perceived control over onset for both T1D and T2D, as expected, attributions for genetics, chance, and other factors typically considered 
low-control were associated with low perceived control among only unaffected respondents. Among affected respondents, these relationships did not follow a discernible pattern. This suggests that perceptions of controllability may be more heavily based on behavioral attributions for these individuals or that other facets of diabetes experience may inform these beliefs. This finding suggests that future work should not rely on the assumption that genetic attributions for diabetes can serve as a surrogate for low control beliefs among individuals with diabetes.

\section{Favorability judgements}

Overall, respondents judged individuals with T1D more favorably than individuals with T2D, consistent with hypotheses. This was true even among individuals with T2D, who rated individuals with T1D almost a full scale point higher in favorability than those with T2D. This is consistent with previous findings in the domain of obesity demonstrating that individuals with obesity engage in in-group stigmatization. ${ }^{32}$

Behavioral causal attributions, such as dietary behavior, were negatively related to the favorability of individuals with T2D and T1D. We did not hypothesize such relationships with regards to T1D, but these results suggest that the link between endorsement of high-fault factors and negative evaluation is likely strong enough that these relationships emerge even when base rates of the attribution are low.

Among respondents with T2D, there was no relationship between genetic causal attributions for T2D and favorability judgements of individuals with T2D, yet attributions for the other non-behavioral factors (germ/virus and chance) were associated with more favorability. This was unexpected given positivity previously seen among individuals with obesity in association with genetic causal attributions for their weight ${ }^{33}$; however, it is consistent with the spotty relationships found between these causal factors and controllability perceptions in the current study. More research in this domain is indicated.

\section{Dimensions of conceptual diabetes knowledge}

Open-ended data provided in response to the diabetes knowledge questions offer insight into how respondent groups consider various dimensions when explaining the differences between T1D and T2D. These responses are likely the most salient features of diabetes for these individuals, linked most closely to respondents' general impressions of the two diabetes types. Over $40 \%$ of unaffected respondents and those with T1D mentioned causal factors in their responses, while only $13 \%$ of respondents with T2D mentioned this dimension. Individuals with T2D may be psychologically motivated not to emphasize aspects of their diagnoses that imply fault and personal responsibility. ${ }^{34}$ Additionally, respondents with T1D and T2D both mentioned treatment elements, pathophysiology, and disease outcomes more often than unaffected individuals. Affected individuals would likely be well educated on these dimensions due to their own life experience. This work suggests that affected and unaffected individuals develop distinct mental models of the illness, its characteristics, and outcomes, likely due to their lived experience.

\section{Limitations}

This study has several limitations. Representation of various demographic features differed by diabetes status and understanding. Differences in group demographics were controlled for in all analyses but still may have influenced data patterns. In part, this is due to demographic differences between ResearchMatch/Facebook patient groups (recruitment sources for affected individuals) and Amazon mTurk (recruitment source for unaffected individuals). mTurk samples have been shown to be very similar to representative US samples ${ }^{35}$; however, the patient population was skewed as is common in clinical research. ${ }^{36}$ In addition, there may be other, more nuanced, measures than favorability that can assess attitudes across T1D and T2D in future work. Finally, associations reported here are cross-sectional and non-directional. As such, the way in which attributions inform attitudes specific to T1D and T2D over time must be investigated further.

\section{CONCLUSIONS}

Until now, the diffuse nature of the literature did not allow for comparisons among different respondent groups and between diabetes types as to causal attributions for diabetes. The current analysis is a step towards understanding how attributions differ, elucidating the reasons for those differences, and considering the influence these differences may have on self-identity, self-efficacy, and perceived treatment response efficacy. These findings also hold implications for clinical and public health diabetes education approaches. To provide a few examples, causal attribution patterns for T1D and T2D are remarkably similar among affected and unaffected groups, making it unlikely that educational materials would need to be differentially tailored to adapt to these patterns. In addition, knowledge results highlight that the high prevalence of T2D is rarely salient to individuals, affected or unaffected and as such may be an area for additional emphasis. Results also demonstrate that incorporating information about genetic factors in T2D in educational materials appears fairly unlikely to lead to fatalistic responses (in that there is low/no relationship between genetic attributions and controllability perceptions), and that this information may additionally be associated with more favorable perceptions of individuals with T2D. In addition to what has been uncovered by the present research, further research in these areas will help inform public health and clinical efforts to prevent and treat T1D and T2D.

Acknowledgements This research was supported by the Intramural Research Program of the National Human Genome Research Institute, National Institutes of Health. The authors would like to thank Corey Miller for his data coding work. 
Contributors SP and KAC conceived of the study. SP, KAC and RWC designed the study. SP, KAC, SEB and MKR analyzed the data. All authors interpreted the data. $\mathrm{SP}$ and MKR drafted the manuscript. All authors contributed substantially to its revision. SP takes responsibility for the paper as a whole.

Funding This work was supported by NHGRI/NIH Z01-HG200383-07.

Competing interests None declared.

Patient consent for publication Not required.

Ethics approval This study was determined to be IRB-exempt by the National Institutes of Health Office of Human Subjects Research.

Provenance and peer review Not commissioned; externally peer reviewed.

Data availability statement Data are available on reasonable request.

Open access This is an open access article distributed in accordance with the Creative Commons Attribution Non Commercial (CC BY-NC 4.0) license, which permits others to distribute, remix, adapt, build upon this work non-commercially, and license their derivative works on different terms, provided the original work is properly cited, appropriate credit is given, any changes made indicated, and the use is non-commercial. See: http://creativecommons.org/licenses/by-nc/4.0/.

\section{REFERENCES}

1. Waters EA, Muff J, Hamilton JG. Multifactorial beliefs about the role of genetics and behavior in common health conditions: prevalence and associations with participant characteristics and engagement in health behaviors. Genet Med 2014;16:913-21.

2. Weiner B, Perry RP, Magnusson J. An attributional analysis of reactions to stigmas. J Pers Soc Psychol 1988;55:738-48.

3. Pociot F, Lernmark Åke. Genetic risk factors for type 1 diabetes. The Lancet 2016;387:2331-9.

4. DiMeglio LA, Evans-Molina C, Oram RA. Type 1 diabetes. Lancet 2018;391:2449-62.

5. Rewers M, Ludvigsson J. Environmental risk factors for type 1 diabetes. Lancet 2016;387:2340-8.

6. Prasad RB, Groop L. Genetics of type 2 diabetes-pitfalls and possibilities. Genes 2015;6:87-123.

7. Willemsen G, Ward KJ, Bell CG, et al. The concordance and heritability of type 2 diabetes in 34,166 twin pairs from international twin registers: the discordant twin (DISCOTWIN) Consortium. Twin Res Hum Genet 2015;18:762-71.

8. Grarup N, Sandholt CH, Hansen T, et al. Genetic susceptibility to type 2 diabetes and obesity: from genome-wide association studies to rare variants and beyond. Diabetologia 2014;57:1528-41.

9. Hu FB, Manson JE, Stampfer MJ, et al. Diet, lifestyle, and the risk of type 2 diabetes mellitus in women. N Engl J Med 2001;345:790-7.

10. Knowler WC, Barrett-Connor E, Fowler SE, et al. Reduction in the incidence of type 2 diabetes with lifestyle intervention or metformin. N Engl J Med 2002;346:393-403.

11. Weiner B. A theory of motivation for some classroom experiences. $J$ Educ Psychol 1979;71:3-25.

12. Corrigan P, Markowitz FE, Watson A, et al. An attribution model of public discrimination towards persons with mental illness. J Health Soc Behav 2003;44:162-79.

13. Kirk SFL, Price SL, Penney TL, et al. Blame, shame, and lack of support: a multilevel study on obesity management. Qual Health Res 2014;24:790-800.

14. Crandall CS, Reser AH. Attributions and Weight-Based Prejudice. In: Brownell KD, Puhl RM, Shwartz MB, et al, eds. Weight bias: nature, consequences, and remedies. New York, NY: Guilford Publications, 2005: p. 83-96.
15. Hoyt CL, Burnette JL, Auster-Gussman L, et al. The obesity stigma asymmetry model: the indirect and divergent effects of blame and changeability beliefs on antifat prejudice. Stigma Health 2017:2:53-65.

16. Browne JL, Ventura A, Mosely K, et al. 'I call it the blame and shame disease': a qualitative study about perceptions of social stigma surrounding type 2 diabetes. BMJ Open 2013;3:e003384.

17. Browne JL, Ventura A, Mosely K, et al. 'I'm not a druggie, I'm just a diabetic': a qualitative study of stigma from the perspective of adults with type 1 diabetes. BMJ Open 2014;4:e005625.

18. Stefanik-Sidener K. Nature, nurture, or that fast food hamburger: media framing of diabetes in the new York times from 2000 to 2010. Health Commun 2013;28:351-8.

19. Sanderson SC, Diefenbach MA, Streicher SA, et al. Genetic and lifestyle causal beliefs about obesity and associated diseases among ethnically diverse patients: a structured interview study. Public Health Genomics 2013;16:83-93.

20. Nguyen AB, Oh A, Moser RP, et al. Perceptions of the roles of behaviour and genetics in disease risk: are they associated with behaviour change attempts. Psychol Health 2015;30:336-53.

21. Daack-Hirsch S, Shah LL, Cady AD. Mental models of cause and inheritance for type 2 diabetes among unaffected individuals who have a positive family history. Qual Health Res 2018;28:534-47.

22. Anderson-Lister G, Treharne GJ. 'Healthy' individuals' perceptions of type 1 and type 2 diabetes cause and management: a 'think-aloud', mixed-methods study using video-based vignettes. $J$ Health Psychol 2014;19:1371-81.

23. Pearl RL, Puhl RM. The distinct effects of internalizing weight bias: an experimental study. Body Image 2016;17:38-42.

24. Livingston JD, Boyd JE. Correlates and consequences of internalized stigma for people living with mental illness: a systematic review and meta-analysis. Soc Sci Med 2010;71:2150-61.

25. Lee RS, Kochman A, Sikkema KJ. Internalized stigma among people living with HIV-AIDS. AIDS Behav 2002;6:309-19.

26. Hornsey MJ, Hogg MA. The effects of status on subgroup relations. Br J Soc Psychol 2002;41:203-18.

27. Moss-Morris R, Weinman J, Petrie K, et al. The revised illness perception questionnaire (IPQ-R). Psychol Health 2002;17:1-16.

28. Persky S, Street RL. Evaluating approaches for communication about genomic influences on body weight. Ann Behav Med 2015;49:675-84.

29. McDonald J. Multiple comparisons. Handbook of biological statistics. 3 ed, 2014.

30. Hornsey MJ, Hogg MA, Similarity I. And subgroup relations: some implications for assimilation. Pers Soc Psychol Bull 2000;26:948-58.

31. Tait KF, Marshall T, Berman J, et al. Clustering of autoimmune disease in parents of siblings from the type 1 diabetes Warren Repository. Diabet Med 2004;21:358-62.

32. Wang SS, Brownell KD, Wadden TA. The influence of the stigma of obesity on overweight individuals. Int J Obes 2004;28:1333-7.

33. Meisel SF, Wardle J. 'Battling my biology': psychological effects of genetic testing for risk of weight gain. J Genet Couns 2014;23:179-86.

34. Keefer LA, Brown M, McGrew SJ, et al. Growth motivation moderates a self-serving attribution bias in the health domain. Pers Individ Dif 2018;134:60-5.

35. Bartneck C, Duenser A, Moltchanova E, et al. Comparing the similarity of responses received from studies in Amazon's mechanical Turk to studies conducted online and with direct recruitment. PLoS One 2015;10:e0121595.

36. Shapiro DN, Chandler J, Mueller PA. Using mechanical Turk to study clinical populations. Clin Psychol Sci 2013;1:213-20. 